\title{
Implicaciones de la educación artística en la salud, bienestar y calidad de vida de los adultos mayores. Una respuesta al envejecimiento activo
}

\section{Implications of artistic education in the health, well-being and quality of life of older people. A response to active aging}

\section{Milena Juliana Castellarin}

Universidad Nacional de Rosario, Argentina.

milenajcastellarin@gmail.com

\section{Lía Macarena Caamaño González \\ Universidad de Chile, Chile. \\ liacaamano@gmail.com}

\section{Resumen:}

Este artículo constituye una reflexión en torno al papel relevante que ofrece la Educación Artística en la tercera edad, tomando como punto de partida nuestra experiencia pedagógica en el desarrollo de talleres de expresión artística destinados a personas adultas mayores. Se pretende identificar y analizar los beneficios que resultan de la práctica creativa y su incidencia en la salud, bienestar y calidad de vida de este grupo etario.

La educación artística se presenta como un espacio propicio de contención donde transitar la vejez, involucrando e impactando positivamente en las esferas biológica, cognitiva, psicológica y social; posicionándose como una alternativa sumamente efectiva para fomentar el envejecimiento activo en la sociedad.

\section{Sugerencias para citar este artículo}

Castellarin, Milena Juliana y Caamaño González, Lía Macarena (2020). Implicaciones de la educación artística en la salud, bienestar y calidad de vida de los adultos mayores. Una respuesta al envejecimiento activo. Tercio Creciente, 17, págs. 7-20.

https://dx.doi.org/10.17561/rtc.n17.1

CASTELLARIN, MILENA JULIANA y CAAMAÑO GONZÁLEZ, MACARENA. Implicaciones de la educación artística en la salud, bienestar y calidad de vida de los adultos mayores. Una respuesta al envejecimiento activo. Tercio Creciente, enero 2020. $\mathrm{n}^{\circ} 17, \mathrm{pp} .7-20$. https://dx.doi.org/10.17561/rtc.n17.1 


\section{Abstract:}

This article constitutes a reflection on the relevant role that Art Education offers to the elders, our starting point is our pedagogical experience in the development of artistic expression classes directed to older people. It is intended to identify and analyze the benefits that result from a creative practice and its impact on health, well-being and the quality of life for this age group.

Artistic education is presented as a conducive space for support where transit to old age, is impacted positively in the biological, cognitive, psychological and social spheres. Art education is an effective alternative to promote active aging in society.

\section{Palabras Clave / Key words}

Adulto mayor, tercera edad, educación artística, creatividad, bienestar, calidad de vida, envejecimiento activo / Elderly, senior citizens, arts education, creativity, well-being, quality of life, active aging.

\section{Sugerencias para citar este artículo}

Castellarin, Milena Juliana y Caamaño González, Macarena (2020). Implicaciones de la educación artística en la salud, bienestar y calidad de vida de los adultos mayores. Una respuesta al envejecimiento activo. Tercio Creciente, 17, págs. 7-20. https://dx.doi.org/10.17561/rtc.n17.1

CASTELLARIN, MILENA JULIANA y CAAMAÑO GONZÁLEZ, MACARENA. Implicaciones de la educación artística en la salud, bienestar y calidad de vida de los adultos mayores. Una respuesta al envejecimiento activo. Tercio Creciente, enero 2020. $\mathrm{n}^{\mathrm{o}} 17$, pp. 7-20. https://dx.doi.org/10.17561/rtc.n17.1 
El presente artículo plantea una reflexión acerca de la importancia que acarrea la práctica de Educación Artística en la tercera edad, basándonos en nuestra experiencia impartiendo talleres de expresión artística para adultos y adultas mayores y rescatando el impacto positivo en su calidad de vida.

La motivación principal que nos condujo a la redacción de este artículo fue haber transitado experiencias como docentes de talleres de expresión artística, destinados a mejorar la salud y calidad de vida de personas adultas mayores. En ambos casos se trató de talleres semanales y grupos mixtos, uno de ellos estuvo a cargo de la Lic. Lia Caamaño y tuvo lugar en la Agrupación de Adultos Mayores de la comuna de Colina, en Santiago de Chile (2014-2018) y el otro desarrollado por la Lic. Milena Juliana Castellarin en un Centro de Día de barrio Fisherton, perteneciente al Instituto de Neurociencias Cognitivas ubicado en la ciudad de Rosario, Argentina (2016-2018) y destinado a beneficiarios de clínica de la memoria.

\section{Introducción}

La Organización Mundial de la Salud considera que al pasar los sesenta años las personas comenzamos a transitar la denominada "tercera edad", de igual manera invitamos a pensar la noción de envejecimiento desde un enfoque integral, entendiéndolo como un proceso cuyas etapas responden a las circunstancias de vida y al desarrollo biológico, cognitivo, psicológico y social de cada persona.

La sociedad atribuye a la vejez un lugar relegado, de descarte, promoviendo incluso mecanismos para disimular las marcas del tiempo a nivel corporal. La imagen que construye en torno a ella es negativa y negadora de esta etapa de la vida como una oportunidad de desenvolverse socialmente, por el contrario, la presenta enfatizando en la disminución de las capacidades y habilidades, agravada por la pérdida de la sexualidad y reducida adaptación a un mundo aceleradamente tecnologizado. Esto afecta al adulto mayor en su autoestima y a quienes no han llegado a esa etapa por el vínculo que ya están generando con la misma.

Nos encontramos con población cada vez más numerosa y longeva, como sociedad nos urge la necesidad de reflexionar en qué casillas estamos ubicando a esta etapa y qué realidades se están presentando a este colectivo. Es momento de quitar a la tercera edad del rincón apartado donde se la ha ubicado tantas veces, asumirla como una etapa del ser humano, ponerle atención y accionar en pos de su calidad de vida.

La representación social de la vejez influye en la autopercepción del adulto mayor y en cómo nos vamos acercando a esa etapa. 

que a unas características fisiológicas, es decir, la vejez empieza cuando un grupo social o sociedad de la que forma parte lo reconoce como una persona vieja, produciéndose una situación en que la edad social que se estipula como determinante del inicio de la vejez nada tiene que ver con la edad cronológica. De alguna manera, son las sociedades las que condicionan la cantidad y calidad de vida en esta etapa y que la situación de dependencia en que se encuentran los ancianos se debe a la política social" (Colom, 1999, p. 52).

Resulta necesario reconfigurar la representación social de la vejez para propagar su valoración positiva y promover la concepción de adultos/as mayores como sujetos activos. Por esta razón resulta indispensable su abordaje como seres sociales, puesto que el rol activo dentro de la comunidad donde está inserto/a conforma un factor determinante en su calidad de vida.

\section{Educación Artística y Tercera Edad}

En esta línea, la Educación Artística en la tercera edad tiene un papel más que relevante ya que la organización del trabajo en grupos dentro del taller se encuentra en directa relación con las necesidades de las personas mayores, promoviendo su rol activo desde la creatividad, la reflexión y el quehacer artístico; generando la conformación de redes, fomentando la creación y sostén de vínculos sociales.

Nuestra práctica de taller encuadrada dentro de la Educación Artística se despega de la concepción plástica academicista, la cual centra al arte en el producto bello acabado, para ubicarlo en el proceso: El arte como actividad experiencial. Ya en 1934 el filósofo John Dewey nos invitaba a abandonar la idea de arte como objeto sublime, lejano y apartado de la cotidianeidad, para pensarlo como "experiencia estética" y vincularlo directamente con la vida, el cuerpo, la actividad y el momento de la creación.

"El producto del arte -templo, pintura, estatua, poema- no es la obra de arte, sino que esta se realiza cuando el ser humano coopera con el producto de modo que su resultado sea una experiencia gozada a causa de sus propiedades liberadoras y ordenadas" (Dewey, 1934, p. 241).

Nuestras propuestas están centradas en las personas con quienes trabajamos, quienes mediante la experiencia artística fortalecen y potencian sus habilidades y capacidades. El proceso creativo parte de su realidad cercana, del contexto social donde trabajamos, de sus vivencias subjetivas y contribuye a la mejora de su calidad de vida. "Las experiencias estéticas son manifestaciones de nuestro potencial para desarrollar una vida mejor, más digna, más inteligente, más justa" (Augustowsky, 2012, p. 14). 
Entendemos la Educación Artística en adultos mayores como una herramienta efectiva para la promoción de la salud, teniendo en cuenta el grado de vulnerabilidad de este grupo y sus necesidades específicas:

"La promoción de la salud se describe como un proceso, cuyo objeto consiste en fortalecer las habilidades y capacidades de las personas para emprender una acción, y la capacidad de los grupos o las comunidades para actuar colectivamente con el fin de ejercer control sobre los determinantes de la salud". (Ministerio de Salud, Gobierno de Chile, 2016, Promoción de Salud)

Entendemos que la promoción de la salud no remite solamente a la ausencia de enfermedad, o la comprensión, prevención y enfrentamiento de los procesos patológicos: Se trata de defender y elevar la calidad y la dignidad de la vida. "La salud es fuerza, sincronía interna, organización, interconexión intelecto-cuerpo-espíritu, ocurriendo aquí y ahora". (Ministerio de Salud, Gobierno de Chile, 2015, Promoción de salud función esencial de Salud Pública).

La Educación Artística en la tercera edad promueve vías de expresión y desarrollo de la creatividad por lo que podría ligarse al concepto de "salutogénesis", que contrariamente al enfoque patogénico (centrado en la enfermedad), trabaja e incide sobre los aspectos sanos y positivos de la persona, centrándose en sus condiciones de vida y entorno, capacitándola y empoderándola, manteniendo un abordaje multidisciplinar. La promoción de la salud es fundamental en adultos y adultas mayores apuntada a un "envejecimiento activo". La posibilidad de participar en un espacio grupal de taller que fomenta la creatividad, contribuye a estimular sus funciones físicas, mentales, emocionales y sociales, promoviendo su capacidad de adaptarse a los cambios propios del envejecimiento, sintiéndose contenidos/as y acompañados/as, posicionándose como sujetos activos y generando los recursos necesarios para serlo.

El taller brinda un espacio donde relacionarse mediante lenguajes artísticos y se convierte en un aporte significativo en la calidad de vida del adulto y adulta mayor, incidiendo directamente sobre los indicadores de bienestar planteados por Carol Ryff (1989-1998) en su Modelo de Bienestar Psicológico; teoría que vincula la calidad de vida al bienestar subjetivo, el cual puede ser medido y a la vez potenciado teniendo en cuenta las siguientes dimensiones de la persona: Autoaceptación, Relaciones positivas con los demás, Autonomía, Dominio del ambiente, Propósito de vida y Crecimiento personal.

Diversos autores vinculan fuertemente el bienestar psicológico del adulto/a mayor a su autoestima, la cual involucra el aspecto emocional y repercute en las distintas áreas de su vida. "Se define generalmente como una actitud o sentimiento positivo o negativo hacia la persona, basada en la evaluación de sus propias características, e incluye sentimientos de satisfacción consigo mismo." (Ortiz y Castro, 2009, p. 27).

Además de la ya mencionada construcción social de la vejez que afecta a la imagen que los/as adultos/as mayores perciben de sí mismos/as, otro factor agravante en la 
disminución de su autoestima es la comparación que suelen realizar entre las habilidades y características propias de su juventud frente a los cambios o disminuciones que puedan experimentar en sus facultades físicas y/o cognitivas propias del envejecimiento, lo cual afecta su autosatisfacción.

Solo dimensionando el impacto que presenta el fortalecimiento de la autoestima en la calidad de vida de los/as adultos/as mayores, podemos comprender la importancia que tiene desarrollar actividades que la favorezcan. El encuentro con pares permite transitar la vejez sintiéndose acompañados por otros/as que están vivenciando la misma etapa, plantear objetivos comunes e involucrarse en proyectos que favorecen su actividad.

La OMS hace referencia al término "envejecimiento activo" como "proceso de optimización de las oportunidades de salud, participación y seguridad con el fin de mejorar la calidad de vida de las personas a medida que envejecen" (OMS, Informe mundial sobre el envejecimiento y la salud, 2015, p. 5). El envejecimiento activo se presenta como un gran desafío en las políticas públicas de muchos países, dado que se encuentra determinado en parte por los aspectos conductuales y personales, pero en gran medida por los factores económicos, sociales, influidos por el entorno físico y en directa relación con los sistemas sanitarios y sociales.

Desde nuestra experiencia docente en el dictado de talleres de expresión artística destinados a tercera edad, destacamos especialmente la eficacia de las herramientas y lenguajes artísticos en la conformación de propuestas que contribuyan al bienestar de los/as adultas mayores, favoreciendo su socialización, su independencia y el mencionado rol activo, contribuyendo a su desarrollo tanto desde la producción creativa personal como desde la creación colectiva en las propuestas grupales.

¿Será entonces el momento de considerar formalmente la potencialidad de la educación artística en la tercera edad?

\section{La importancia del grupo}

Observando que la tercera edad viene acompañada en ocasiones de cambios drásticos en los hábitos de vida y que muchos/as mayores afrontan el riesgo de exclusión social, presentamos el taller de expresión artística como una alternativa para prevenir y aplacar esta problemática.

Entendemos a la creación de este tipo de talleres como una forma de propiciar el cumplimiento de derechos fundamentales para las personas adultas mayores, así como lo estipula la ONU en los "Principios de las Naciones Unidas en favor de las Personas de Edad" (1991): "Las personas de edad deberán poder aprovechar las oportunidades para desarrollar plenamente su potencial" (derecho nro. 15) y "Las personas de edad deberán tener acceso 
a los recursos educativos, culturales, espirituales y recreativos de la sociedad"(derecho nro. 16), reafirmándolo años más tarde en la "Declaración Política y Plan de Acción Internacional de Madrid sobre el Envejecimiento" (2002), estableciendo como medida de prevención: "Promover la participación de las personas de edad en actividades cívicas y culturales como estrategia para luchar contra el aislamiento social y favorecer su habilitación".

Planteamos propuestas constructivas que vinculan a la persona mayor con el medio que le rodea, partimos de su realidad para llevar adelante una actividad artística que la involucra con su entorno, ayudándole a generar pertenencia a su comunidad, a que sostenga el contacto con el medio que le rodea y que además se sienta útil, valorada y aceptada por otros/as integrantes de la sociedad.

\footnotetext{
"La comunidad es también el escenario de la construcción de relaciones humanas y valores interpersonales vinculados al conocimiento de los y las demás, a la conciencia del colectivo y de una situación de vida compartida, así como a la búsqueda de cambio social a través de la organización y la cohesión social" (Montero, 2004; Sánchez, 2007)." (Cueto, Espinosa, Guillén, y Seminario, 2014, p. 2).
}

La comunidad es el espacio que se crea tanto física como emocionalmente, es el momento y lugar del taller, las personas que lo conforman, las herramientas que comparten, las relaciones interpersonales, las sensaciones y emociones que afloran, las conversaciones mientras trabajan, el currículum oculto. También invitamos a pensar las comunidades como "fuerzas preventivas" (Maguire,1980) que asisten a sus miembros cuando se presenta alguna adversidad, convirtiéndose en verdaderos tejidos de apoyo. A esto atribuimos la importancia de destacar que a través del trabajo del taller de expresión artística se potencia de forma intrínseca la generación de redes, la intensificación de la cohesión social y el vínculo grupal.

Desde la configuración como grupo y su identificación con éste, se comienza a generar sentido de pertenencia, posicionándose un yo personal y a la vez colectivo, compartiendo intereses y experiencias, dando pie al sentido de comunidad, el cual se define como "sentimiento de pertenencia a una comunidad, así como la percepción de interdependencia entre sus miembros, por la cual cada miembro del colectivo se siente importante para los demás y para el grupo (Sánchez, 2007). De este modo, el sentido de comunidad favorece el surgimiento de un compromiso conjunto por la satisfacción de las necesidades grupales, psicológicas y materiales (McMillan \& Chavis, 1986)." (Cueto, Espinosa, Guillén, y Seminario, 2014, p. 2).

En el taller se derriba el aislamiento social que suele afectar a la tercera edad, reuniéndose en un espacio de pertenencia, en un grupo propio donde entablar vínculos y generar redes de apoyo:

"De esta manera, realizando propuestas de trabajo concretas, adaptadas a los mayores, que favorezcan su implicación y que les resulten motivadoras, estamos 
potenciando su funcionamiento cognitivo y previniendo en gran medida el deterioro. Pero estas intervenciones también tienen un fin lúdico, de diversión y socialización con otras personas, fomentando el desarrollo socio-emocional. De este modo se consigue que el anciano continúe activo a nivel físico, cognitivo, social y afectivo, mejorando su calidad de vida y promoviendo un envejecimiento saludable (Zamarrón, 2007)." (Carrascal y Solera, 2014, p. 13)

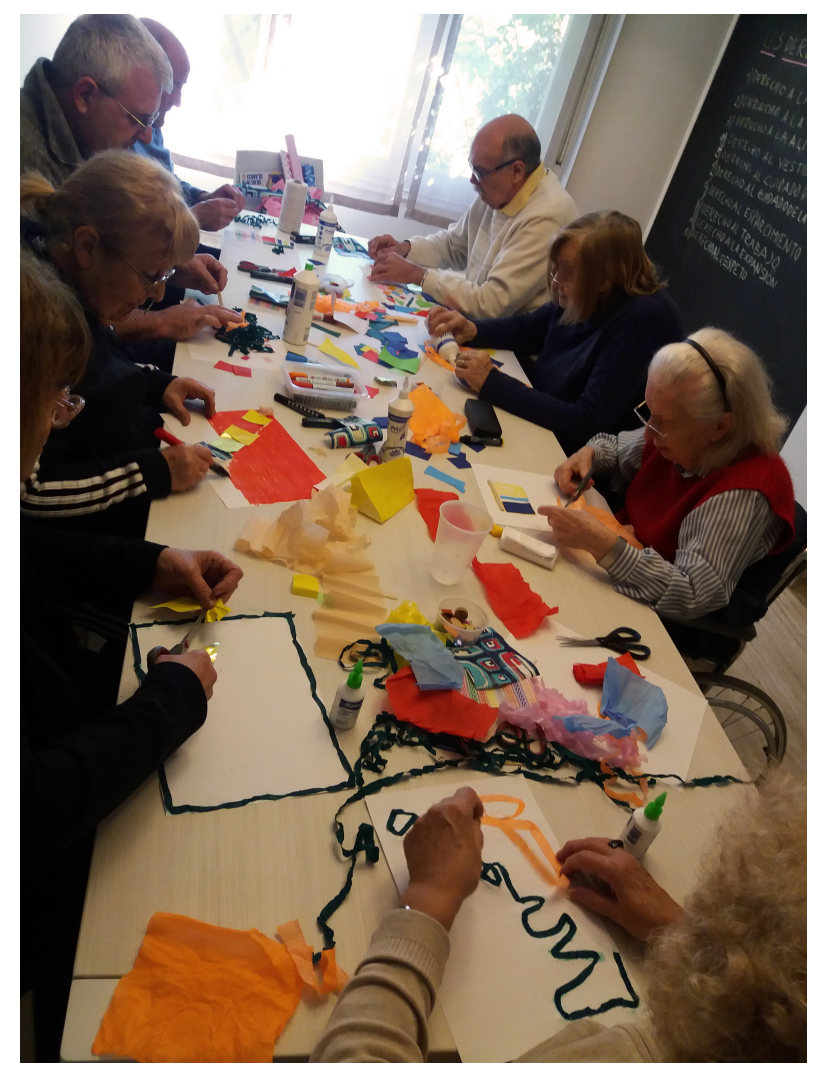

Fotografía 1: Registro visual de una tarde de Taller (Rosario, Argentina)

El taller de arte no está pensado como espacio aislado dentro de la vida de los/as adultos/as mayores sino como un aporte a la calidad de la misma; desde el bienestar colectivo afianzando lazos y conformando comunidad, y para su bienestar subjetivo (individual), siendo fundamental el desarrollo de la creatividad para su envejecimiento activo, posibilitando la gestión emocional, así como impacto positivo en su manera de abordar la realidad y de percibirla.

Las "relaciones positivas con los/as otros/as", la posibilidad de sostener vínculos satisfactorios, mantener apertura y desarrollar empatía, y el "dominio del entorno" no solo pensado como capacidad de adaptación sino también como "capacidad individual para crear 
o elegir ambientes favorables para satisfacer los deseos y necesidades propias", son dos aspectos fundamentales para alcanzar el bienestar psicológico (Carol Ryff) y el encuentro artístico los fomenta ampliamente.

\section{Continuar Aprendiendo en la vejez}

Frente al sentimiento de pérdida de libertad que suelen experimentar las personas mayores que se encuentran a cargo de otros familiares, el taller de arte representa ese espacio donde aún pueden tomar decisiones, contribuyendo a su independencia, ampliando su capacidad resolutiva frente a los problemas y empoderamiento.

La imagen social de la vejez que ya hemos mencionado no asocia el aprendizaje a la tercera edad sino a la infancia y a la juventud, colocando a la vejez en el lugar de una etapa sin novedad y carente de desarrollo intelectual. La Educación Artística brinda a la persona mayor la posibilidad de romper con esa barrera, de participar activamente en un espacio de taller donde vincularse creativamente, retomar la actividad productiva, trabajar sobre la propia identidad y reestablecer relaciones afectivas, en un ambiente de contención y seguridad.

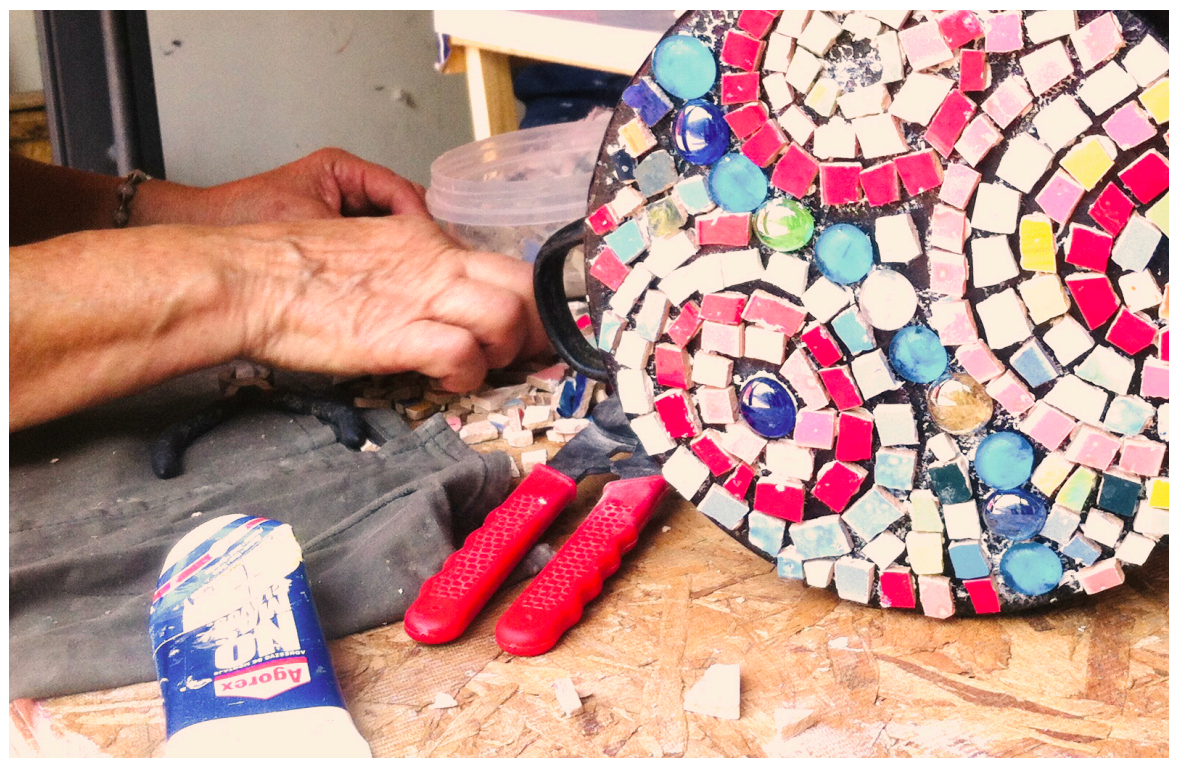

Fotografía 2: Registro visual de adulta mayor trabajando en la técnica del mosaico (Colina, Santiago de Chile). 
Los lenguajes artísticos poseen un gran potencial que debemos saber aprovechar para estimular en los/as adultos/as mayores el deseo de aprender, despertar su curiosidad y encender nuevamente su motivación.

El taller de arte es un espacio de aprendizaje y creación que plantea a los/as adultos/as mayores nuevos desafíos; separándolos de la pasividad y de la idea de inutilidad que a muchos acecha y contribuyendo directamente al fortalecimiento de su autoestima, al ser conscientes de que son capaces de aprender cosas nuevas y crear a partir de estas, sentirse valiosos y percibir esta valoración en su entorno (familiares, amistades, compañeros/as).

El saberse capaces los conduce al empoderamiento, que también se da a nivel colectivo fruto de la cohesión del grupo y así comienzan a surgir propuestas espontáneas que van desde su organización en pequeños proyectos hasta el desarrollo de una exposición de arte para mostrar a los/las demás su producción artística, postulaciones para desarrollar iniciativas en su beneficio, conformación de clubes del adulto mayor, etc. Resulta fundamental destacar la relevancia del empoderamiento colectivo en el desarrollo de un envejecimiento activo, saludable y con propósito, y el rol distinguido que la Educación Artística puede ejercer dentro del mismo.

La extensión de la etapa de aprendizaje coopera con otras de las dimensiones planteadas para alcanzar el bienestar psicológico (Carol Ryff), siendo el "propósito de vida" y "crecimiento personal" nociones de gran relevancia en la tercera edad. Resulta sumamente necesario que las personas mayores se propongan objetivos y metas, encuentren sentido a sus vidas, continúen abiertos a experiencias nuevas y desafiantes, expandiendo sus potencialidades, descubriendo sus habilidades a través del quehacer artístico y el espacio creativo del taller, adquiriendo seguridad y los recursos para seguir avanzando.

Atrás quedó la noción de "artista genio" recluido, apartado, de la cual hemos heredado el concepto elitista de creatividad entendida como una cualidad individual, escasa y especial, que solo algunos iluminados poseen. Desde nuestras propuestas de educación artística destinadas a tercera edad hemos extendido la perspectiva de "creatividad horizontal" (Julio Romero, 2010), como una capacidad inherente al ser humano con posibilidad de ser expandida y también la "creatividad extendida" como modo de ser, pensar y hacer, frente a esa idea de "golpe de inspiración" que aún suele tener vigencia. Por eso consideramos fundamental facilitar las condiciones de participación de las personas mayores como agentes activos en procesos de creación, su rol dentro del taller como sujetos creativos y no sólo como observadores de la creatividad ajena.

Un paso más allá, Julio Romero (2010) quien categoriza diferentes tipos de creatividad, nos presenta la "creatividad distribuida" refiriéndose a "las posibilidades de creación y de participación como creadores en proyectos compartidos y utilizando sus diferentes saberes y capacidades, así como los materiales, instrumentos y herramientas al alcance, según las diferentes situaciones y posibilidades de las personas. Si hablamos de creatividad distribuida, la creatividad no está en la persona, está en la relación que se puede 
establecer entre las personas, los saberes, los materiales, las herramientas e instrumentos de la cultura, interaccionando en procesos de colaboración. Según esto, la creatividad no se tiene, se comparte; la participación en proyectos creativos compartidos, por tanto, es una clave fundamental" (Romero, 2010, p. 97). Abrazamos la creatividad compartida y pensamos también en su concepto de "creatividad transformadora" la cual "transforma, no ya los objetos o los materiales, sino las personas, las comunidades, la cultura, el modo de ver y verse en la realidad, y a ésta misma".

El proceso creativo transforma a las personas que intervienen en él, modifica sus relaciones y estimula las funciones físicas, mentales, emocionales y sociales. La creatividad no se restringe solo a la producción plástica dentro del taller de arte, se extiende en la vida y la favorece.

En el taller de arte se involucra el cuerpo incentivando la propia escucha, el autocuidado y trabajando la motricidad fina, la coordinación y el movimiento; se activan las funciones cognitivas, contribuyendo a la memoria, la atención y la concentración; se estimula la imaginación, la espontaneidad, el control de la ansiedad y la paciencia; se posibilita el pensamiento crítico, la reflexión, la elaboración de opiniones, el sentido de orientación y la toma de decisiones. Se favorece la neuroplasticidad, desarrollando nuevos aprendizajes, permitiéndoles sentirse capaces:

"A pesar del deterioro físico y cognitivo a edades adultas, conservamos la mayoría de las células cerebrales hasta el final de nuestra vida, por lo que si seguimos utilizando nuestro cerebro de forma activa seremos capaces de construir caminos neuronales. Esta estimulación cognitiva, además de potenciar un continuo proceso creativo, ayuda a que las personas intenten esforzarse al máximo, adaptándose a su entorno y modelando su vida (Fischbach y Fischbach, 2005; Robinson y Aronia, 2004)." (Carrascal y Solera, 2014, p. 12).

Con el pasar de las clases descubrimos que las personas mayores esperan con ansias el día del taller de arte y que su autoestima va en mejora al sentir satisfacción por aquello que son capaces de hacer, al compartir un espacio del que se sienten parte, al lograr aprender cosas nuevas, tener experiencias significativas, explorar propuestas creativas y trabajar con materiales que les resultan motivadores.

\section{Conclusión}

A modo de conclusión, consideramos que apremia implicarse en promover el bienestar de nuestros/as adultos/as mayores, aún más cuando existen proyecciones de aumento de este grupo etario, por tanto, rescatar las aportaciones de las diversas disciplinas debería ser una tarea primaria común y el accionar en esta causa, una medida efectiva y urgente. 
Desde nuestro lugar proponemos y exponemos el valor del ejercicio creativo en comunidad, como una forma de vivir y hacer concreto el envejecimiento activo, que si bien ha sido instaurado como un lineamiento internacional, en muchos países se encuentra lejos de ser una realidad.

Conocer los beneficios que aporta la educación artística a las personas adultas mayores, y cómo implica las distintas áreas del ser, nos permite valorarla como herramienta y valerse de sus cualidades para lograr una mejor calidad de vida en la vejez.

Finalmente, deseando haber motivado el interés de otros/as profesionales del área, esperando que puedan descubrir las potencialidades de la educación artística y considerarla formalmente en la implementación de programas de fomento del envejecimiento activo; alentamos a la creación de espacios de encuentro a través de las artes dentro de las instituciones que trabajan con adultos atravesando su vejez. 
Augustowsky, Gabriela. (2012). El arte en la enseñanza. Buenos Aires, Argentina: Paidós

Carrascal, Silvia y Solera, Eva. (2014). Creatividad y desarrollo cognitivo en personas mayores. Arte, Individuo y Sociedad, 26(1), 9-19. Recuperar información en: https://scielo.conicyt.cl/scielo.php?script=sci arttext\&pid=S071822282016000100004. https://doi.org/10.5209/rev ARIS.2014.v26.n1.40100

Colom Bauzá, Joana. (1999). Vejez, representación social y roles de género. Educació i Cultura 12, 47-56. Recuperar información en: http://ibdigital.uib.es/greenstone/collect/educacio/index/assoc/Educacio/ i Cultu/ra 1999 v/12p047.dir/Educacio i Cultura 1999v12p047.pdf.

Cueto, Rosa María; Espinosa, Agustín; Guillén, Henry y Seminario, Miguel (2014). Sentido de Comunidad Como Fuente de Bienestar en Poblaciones Socialmente Vulnerables de Lima, Perú. Psykhe, 25(1), 1-18. Recuperar información en: https://scielo.conicyt.cl/scielo.php?script=sci arttext\&pid=S071822282016000100004. https://doi.org/10.7764/psykhe.25.1.814

Dewey, John. (1934). El arte como experiencia. Barcelona, España: Paidós.

Ministerio de Salud, Gobierno de Chile. (2015) Promoción de salud función esencial de Salud Pública. Recuperado de: https://www.minsal.cl/sites/default/files/PROMOCION SALUD FUNCION SALUD P UBLICA.pdf

Ministerio de Salud, Gobierno de Chile. (2016) Promoción de Salud. Recuperado de https://www.minsal.cl/wp-content/uploads/2016/09/1 PROMOCION-DE-SALUD.pdf

Organización de las Naciones Unidas. (1991). Principios de las Naciones Unidas en favor de las Personas de Edad. Recuperado de https://undocs.org/es/A/RES/46/91

Organización de las Naciones Unidas. (2002). Declaración Política y Plan de Acción Internacional de Madrid sobre el Envejecimiento. Recuperado de: https://www.un.org/esa/socdev/documents/ageing/MIPAA/political-declaration-sp.pdf

Organización Mundial de la Salud. (2015). Informe mundial sobre el envejecimiento y la salud. Recuperado de https://apps.who.int/iris/bitstream/handle/10665/186466/9789240694873 spa.pdf;jsessioni $\mathrm{d}=\mathrm{D} 118 \mathrm{BAF} 27 \mathrm{~A} 5 \mathrm{BA} 86977162636 \mathrm{A7444AE7? \text {sequence } = 1}$ 
Ortiz Arriagada, Juana Beatriz y Castro Salas, Manuel. (2009). Bienestar psicológico de los adultos mayores, su relación con la autoestima y la autoeficacia. Contribución de enfermería. Ciencia y Enfermería XV (1): 25-31. Recuperado de https://scielo.conicyt.cl/scielo.php?script=sci arttext\&pid=S071795532009000100004. https://doi.org/10.4067/S0717-95532009000100004

Romero, Julio. (2010). Creatividad distribuida y otros apoyos para la educación creadora. Pulso. Revista de Educación, 33, 87-107. Recuperado de: https://pdfs.semanticscholar.org/f7aa/7c5f4ca331e74e14cde179d428bcee72ec8a.pdf

Villar, Camila. (2016). Líneas de expresión una experiencia de Arte terapia con Adultas Mayores en contexto comunitario (Monografía para optar al título de especialista en Terapias de Arte, mención Arte terapia.). Universidad de Chile, Santiago, Chile. 20 